\title{
Implementation and Analytical Model of Three-Phase Four-Switch Power Factor Corrector
}

\author{
Jiri Klima \\ Department of Electrical Engineering and Automation \\ Technical Faculty of CZU in Prague \\ 16627 Prague 6, Czech Republic \\ E-mail: klima@tt.czu.cz
}

\begin{abstract}
This paper presents a three-phase fourswitch converter aimed at compensating reactive power in case of distorted and unbalanced source voltages. An original closed-form solution of line currents, based on the mixed p-z approach is presented. The analytical and experimental results are provided to verify the effectiveness of the proposed control scheme.
\end{abstract}

\section{Key words}

SV PWM, Four-Switch Converter, AC-DC Power Converters, Power Factor Correction

\section{Introduction}

AC-DC conversion is used more and more in a wide field of applications. A great attention has therefore been focused recently on the research and development of pulse width modulated rectifiers (PWM rectifiers) [1], [2].

It is well known that a three-phase converter can develop various faults. The interruption of the manufacturing or other process due to a PWM rectifier fault would result in financial losses or even in some serious damage. That is why improved reliability, and fault tolerant operation of such systems are welcomed today. In general, when one of these faults occur, the isolation of the fault is preferable, keeping the system in partial operation, instead of shutting down the whole process.

The converter topology and operation modes of the component minimized three-phase PWM rectifier supplied from unbalanced source voltage are analysed in the paper as well as the novel strategy of the SVM suggested. Finally, an original closed-form solution of line currents, based on the mixed p-z approach [3], [4], [5] is introduced. The results of simulation and tests done on the component minimized three-phase PWM rectifier connected at the three-phase unsymmetrical mains are shown as well.

\author{
Jiri Skramlik, Viktor Valouch
}

Institute of Thermomechanics Academy of Sciences of the Czech Republic Dolejškova 5, 18202 Praha 8, Czech Republic E-mail: valouch@,iee.cas.cz

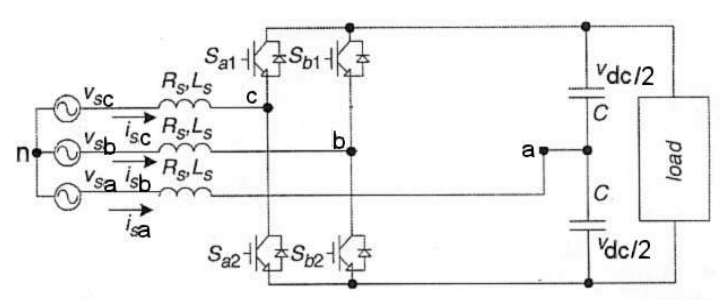

Fig.1 Three-phase four-switch rectifier

2. Topology of converter and mode of operation

A circuit diagram of the four-switch rectifier is shown in Fig.1. Three inductors are used to achieve line current filtering and to obtain dc link voltage boost operation. The scheme employs four switches $S_{a 1}, S_{a 2}$, $\mathrm{S}_{\mathrm{b} 1}$, and $\mathrm{S}_{\mathrm{b} 2}$ and four diodes to generate output voltage. The phase "a" is directly connected to the midpoint of the split dc capacitor.

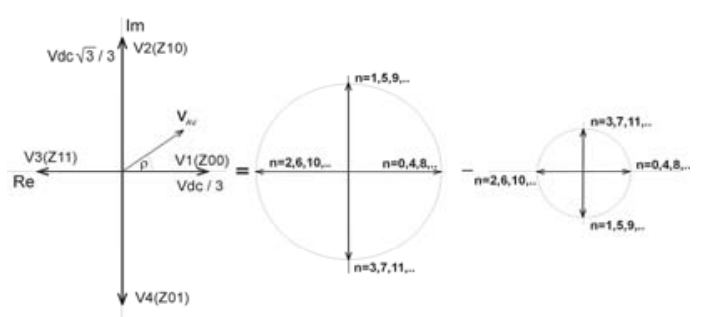

Fig.2 Voltage vector decomposition into two symmetrical sequences: positive $V_{P}(n)$ and negative $\mathrm{V}_{\mathrm{N}}(\mathrm{n})$.

From the combination of the conduction states of the power switches originate four different vectors in the $\alpha \beta$ plane as shown in Fig.2. These vectors are $\pi / 2$ away from each other. Additionally, these vectors do not have the same amplitude as in the classical B6 connection. Vectors lying in the real axis have the amplitude of $\mathrm{V}_{\mathrm{dc}} / 3$, whereas the vectors lying in the imaginary axis have the amplitude of $\sqrt{3} \mathrm{~V}_{\mathrm{dc}} / 3$. Let us define the per unite time 
$\frac{\mathrm{t}}{\mathrm{T}}=\mathrm{n}+\varepsilon, \quad \mathrm{n}=0,1,2, . ., \quad 0<\varepsilon \leq 1$

(1)

where $\mathrm{T}$ is a period of the modulation (period of the symmetry) and is next called a sector, $\varepsilon$ is per unit time inside of sector.

The voltage vector $\mathrm{V}(\mathrm{n})$ can be expressed by means of the discrete Fourier transform in the symmetrical form as follows from [3]:

$V(n)=\frac{1}{N} \sum_{m=0}^{N-1} V(m) \sum_{i=0}^{N-1} e^{j i(n-m)} \frac{2 \pi}{N}$

where $\mathrm{N}$ is the number of the vectors used per output period and $\mathrm{n}$ is a variable (number of the sector).

For the voltage vectors with $\pi / 2$ symmetry $(\mathrm{N}=4)$ we get:

$V(n)=\frac{V_{d c}(1+\sqrt{3})}{6} e^{j n \frac{\pi}{2}}+\frac{V_{d c}(1-\sqrt{3})}{6} e^{-j n \frac{\pi}{2}}=$

$=V_{P}(n)+V_{N}(n)$

If we use only four voltage vectors, this unsymmetrical sequence (2) can be decomposed into two symmetrical ones (3). The first sequence has a positive direction of rotation with the amplitude $\frac{\mathrm{V}_{\mathrm{dc}}(1+\sqrt{3})}{6}$ and the second sequence has a negative direction of rotation with amplitude $\frac{\mathrm{V}_{\mathrm{dc}}(1-\sqrt{3})}{6}$.

These negative sequences can be used for elimination of the unbalance input voltage. The situation is shown in Fig. 2 .On the left side we can see unsymmetrical sequence of the four-switch converter. The reference voltage $\mathrm{V}_{\mathrm{AV}}$ has a polar angle $\rho$ to the real axes.

\section{Space-vector PWM}

To control the transient responses of the line currents $i_{\mathrm{Sa}}, i_{\mathrm{Sb}}, \quad i_{\mathrm{Sc}}$ precisely, the four-switch rectifier in Fig. 1 has to be operated as a Pulse Width Modulated (PWM) rectifier. By using a proper PWM strategy, the line currents will be able to follow their reference values with small errors. The higher the switching frequency will be applied, the lower the errors will appear. The line currents are determined by the voltage drops at the ac inductors $\left(L_{\mathrm{S}}, R_{\mathrm{S}}\right)$. If the line voltage vector $v_{\mathrm{S}}$ is measured, the reference of the rectifier ac-terminal voltage vector $v^{*}$ may be calculated by using the inductor parameters $L_{\mathrm{S}}, R_{\mathrm{S}}$ and the reference of the line current vector $i_{\mathrm{S}}^{*}$ as follows:

$$
\boldsymbol{v}^{*}=\boldsymbol{v}_{\mathrm{S}}-\left(R_{\mathrm{S}}+L_{\mathrm{S}} \frac{\mathrm{d}}{\mathrm{d} t}\right) \boldsymbol{i}_{\mathrm{S}}^{*}
$$

We have proposed a new Space Vector Modulation (SVM) strategy for the three-phase four-switch rectifier in Fig.1.

\section{Closed-form solution}

If the four-switch rectifier is operated as the PWM rectifier with the SVM strategy mentioned in the previous chapter, expression (3) must be modified to respect the switching intervals. If $\varepsilon$ (p.u.) is the time inside every sector, the voltage vector may be defined as follows [5]:

$$
\begin{aligned}
& \boldsymbol{V}(n, \varepsilon)=\boldsymbol{V}_{P}(n, \varepsilon)+\boldsymbol{V}_{N}(n, \varepsilon)= \\
& \sum_{k=1}^{3 N_{1}} \sum_{x=1}^{2} V_{d c}(1+\sqrt{3}) \frac{1}{6} f(\varepsilon, k, x) e^{j n \pi} e^{j \frac{\pi}{2} \alpha(k)} e^{j \pi \frac{(x-1)}{2}}+ \\
& +\sum_{k=1}^{3 N_{1}} \sum_{x=1}^{2} V_{d c}(1-\sqrt{3}) \frac{1}{6} f(\varepsilon, k, x) e^{-j n \pi} e^{-j \frac{\pi}{2} \alpha(k)} e^{-j \pi \frac{(x-1)}{2}}
\end{aligned}
$$

With regard to the SVM strategy mentioned, we get after using the Laplace transform

$$
\begin{aligned}
& \mathrm{i}_{\mathrm{S}}{ }^{*}(\mathrm{p})= \\
& \frac{1}{R_{S}+p_{S}}\left(\frac{V_{m P} e^{j \varphi_{p}}}{p-j \omega}-\frac{V_{m N} e^{j \varphi_{N}}}{p+j \omega}\right)+
\end{aligned}
$$

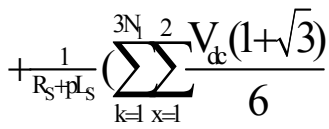

$$
\begin{aligned}
& \left.\frac{e^{p T}}{\left(e^{p T}-e^{j \pi}\right)} e^{j(x-1) \frac{\pi}{2}} e^{j \frac{\pi}{2} \alpha(k)}\left(e^{-p T \varepsilon A, k, x}-e^{-p T \varepsilon_{B, k, x}}\right)\right)+
\end{aligned}
$$

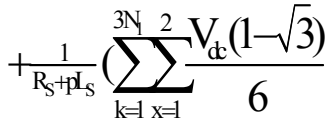

$$
\begin{aligned}
& \left.\frac{e^{p T}}{\left(e^{p T}-e^{-j \pi}\right)} e^{-j(x-1) \frac{\pi}{2}} e^{-j-\frac{\pi}{2} \alpha(k)}\left(e^{-p T \varepsilon_{A, k, x}}-e^{-p \Gamma \varepsilon_{B, k, x}}\right)\right)
\end{aligned}
$$

where $\mathrm{k}=1,2, . .3 \mathrm{~N}_{1}$ and $\mathrm{x}=1,2$ 
The solution in the time domain contains both the steady-state and the transient parts. As our attention is the steady-state solution we can get for $n \rightarrow \infty$ the closed-form solution.

\section{Analytical and experimental results}

To confirm the validity and effectiveness of the proposed analytical method, the closed form analytical and experimental results are presented.

To compare also the influence of the high frequency ripple in the phase currents, Fig. 3 shows the simulated phase current (in p.u.) with its ripple waveform.

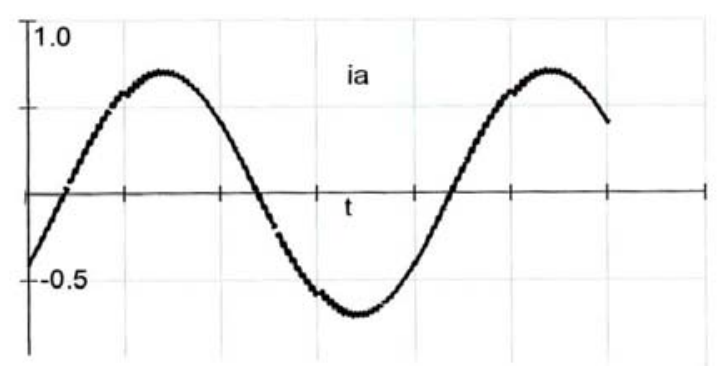

Fig.3 One period of the phase line current

The detail of one period of the measured line current $i_{\text {Sa }}$ and its harmonic spectrum is presented in Fig. 4.

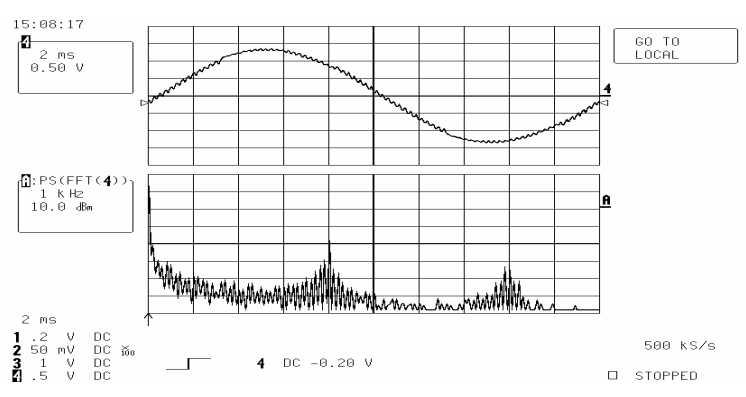

Fig. 4 Experimental results. Detail of one period and harmonic spectrum of line current $i_{\mathrm{Sa}}$

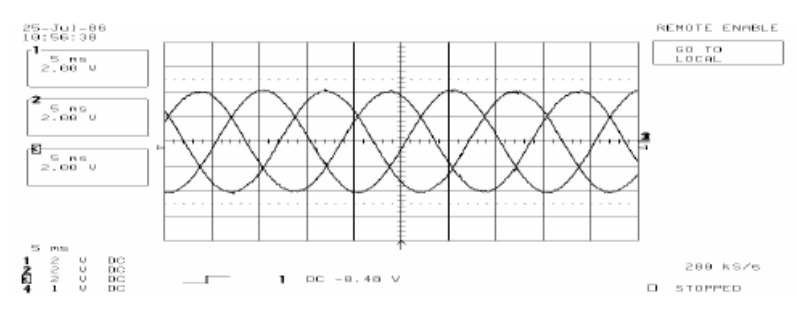

Fig. 5 Experimental results. Three phase line currents

$$
i_{\mathrm{Sa}}, i_{\mathrm{Sb}}, i_{\mathrm{Sc}}
$$

Fig. 5 shows captured time responses of all three line currents $i s a$, isb, isc. From Fig.4 we can see that phase currents are symmetrical and in high correlation with analytical results in Fig 3.

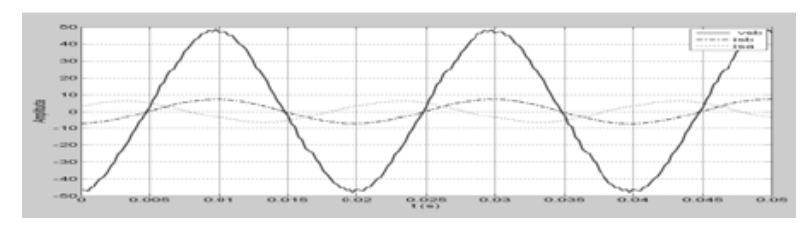

Fig.6 Experimental results. Grid phase voltage, and two line currents

In Fig. 6 we can see the time responses of the source voltage with the line current, in the phase "b", together with the current in the phase "a". The responses have been reconstructed from the data captured and used by the controller. It is evident that the line voltage and current are actually in the phase.

\section{Conclusion}

A three-phase four-switch converter based on a new space-vector PWM strategy is analytically investigated. The scheme with a reduced number of power switches, improving the rectifier efficiency, can compensate the unbalanced source voltages. The analytical results and experiment tests on the component minimized threephase PWM rectifier connected at the three-phase unsymmetrical mains are shown as well to demonstrate the validity and effectiveness of the proposed control scheme.

\section{References}

[1] Ohnishi, T., Okitsu, H.: A Novel PWM Technique for Three Phase Inverter/Converter. Proc. Of Int. Conf. IPEC'83, Tokyo, Japan, 1983, pp. 384395

[2] Malinowski, M., Kazmierkowski, M.P.: Control of Three-Phase PWM Rectifiers. In Kazmierkowski, P.M., Krishnan, R., Blaabjerg, F.: Control in Power Electronics. Academic Press, Elsevier Science (USA), 2002.

[3] Klima,J.: Analytical closed-form solution of a space-vector modulated VSI feeding an induction motor drive.IEEE Transaction on Energy Corversion,vol.17,No2,June 2002,pp.191-196

[4] Klima,J: Mixed p-z approach for time-domain analysis of voltage source inverters with periodic pulsewidth modulation. IEEE Transaction on Circuits 
and Systems II. 2004, No.10, October,ISSN 10577130,pp.529-536

[5] Klima,J.: Time and frequency domain analysis of a fault-tolerant space vector PWM VSI-fed induction motor drive.IEE Proceedings-Electric Power Applications,Vol.152,No4,2005, ISBN 13502352,pp.765-775

\section{Acknowledgement}

The financial supports of the Academy of Sciences of the Czech Republic (Institutional Research Plan Z20570509 of the Institute of Thermomechanics) and of the Grant Agency of the Academy of Sciences of the Czech Republic (Project No. IAA200760703) are highly acknowledged. 